\title{
Aspectos biológicos de Harmonia axyridis alimentada com duas espécies de presas e predação intraguilda com Eriopis connexa
}

\author{
Natália Ribeiro Pereira dos Santos ${ }^{(1)}$, Terezinha Monteiro dos Santos-Cividanes ${ }^{(1)}$, Francisco Jorge Cividanes ${ }^{(2)}$, \\ Anna Carolina Ribeiro dos Anjos $^{(3)}$ e Lelis Vaz Leite de Oliveira ${ }^{(4)}$ \\ ${ }^{(1)}$ Agência Paulista de Tecnologia dos Agronegócios, Avenida Bandeirantes, № 2.419, Vila Virgínia, CEP 14.030-670 Ribeirão Preto, SP, Brasil. \\ E-mail: nati.rps@ig.com.br, terezinha@apta.sp.gov.br (2)Universidade Estadual Paulista, Faculdade de Ciências Agrárias e Veterinárias, \\ Departamento de Fitossanidade, Via de Acesso Prof. Paulo Donato Castellane, s/no, CEP 14884-900 Jaboticabal, SP. E-mail: fjcivida@fcav.unesp.br \\ ${ }^{(3)}$ Agro Energia Santa Luzia Ltda., Caixa Postal 03, CEP 79140-000 Nova Alvorada do Sul, MS. E-mail: anninha bio@yahoo.com.br \\ (4) Universidade Federal de Mato Grosso do Sul, Avenida Costa e Silva, s/no, Cidade Universitária, CEP 79070-900 Campo Grande, MS. \\ E-mail: lelis_vaz_oliveira@yahoo.com.br
}

Resumo - O objetivo deste trabalho foi determinar os aspectos biológicos de Harmonia axyridis, alimentada com duas espécies de presas, e a ocorrência de predação intraguilda com Eriopis connexa. Larvas de H. axyridis foram alimentadas diariamente com ovos de Anagasta kuehniella ou com o pulgão Schizaphis graminum. Adultos da joaninha foram separados em dez casais que receberam o mesmo tipo de alimento da fase larval. Na avaliação da predação, uma larva de quarto instar de cada espécie foi mantida na presença ou ausência de abrigo e de ovos de A. kuehniella. A fase larval de H. axyridis durou 10,2 e 8,9 dias, quando alimentada com A. kuehniella e S. graminum, respectivamente. A sobrevivência do predador, em fase imatura, variou de 70 a $100 \%$. A joaninha apresentou período de oviposição de 47,3 e 51,7 dias, com 887,6 e 822,5 ovos, ao se alimentar de A. kuehniella e S. graminum, respectivamente. A longevidade das fêmeas foi de 74,1 e 76,2 dias e a dos machos de 67,3 e 70,3 dias, em A. kuehniella e S. graminum, respectivamente. H. axyridis atuou como predador intraguilda e foi a espécie dominante na competição com E. connexa.

Termos para indexação: Coccinellidae, Coleoptera, controle biológico.

\section{Biological aspects of Harmonia axyridis fed on two prey species and intraguild predation with Eriopis connexa}

\begin{abstract}
The objective of this work was to determine the biological aspects of Harmonia axyridis, fed on two prey species, and the occurrence of intraguild predation with Eriopis connexa. Larvae of $H$. axyridis were fed daily with Anagasta kuehniella eggs or Schizaphis graminum aphid. Adults of $H$. axyridis were separated in ten couples which received the same food type of the larval stage. For the estimation of predation, a fourth instar larva of each species was maintained in the presence or absence of shelter and eggs of A. kuehniella. The larval stage lasted 10.2 days, when $H$. axyridis fed on A. kuehniella eggs, and 8.9 days when reared with S. graminum. The survival of predator during immature stages ranged from 70 to $100 \%$. H. axyridis had an oviposition period of 47.3 and 51.7 days, laying a total of 887.6 and 822.5 eggs, when fed on A. kuehniella and S. graminum, respectively. Females had longevity of 74.1 and 76.2 days, and males of 67.3 and 70.3 days, fed on A. kuehniella and S. graminum, respectively. H. axyridis acted as an intraguild predator and was the dominant species in the competition with E. connexa.
\end{abstract}

Index terms: Coccinellidae, Coleoptera, biological control.

\section{Introdução}

A joaninha-asiática Harmonia axyridis Pallas, 1773 (Coleoptera: Coccinellidae) é considerada uma predadora voraz e eficaz, principalmente de pulgões, e tem sido utilizada como agente de controle biológico em várias culturas, na América do Norte e Europa (Brown \& Miller, 1998; Michaud, 2000; Kuroda \& Miura, 2003; Landis et al., 2004; Berkvens et al., 2008b).
Em razão do hábito polífago dessa joaninha, os seus aspectos biológicos têm sido determinados sobre diversas espécies de presas. Larvas desse coccinelídeo, quando alimentadas com os pulgões Aphis spiraecola Patch e Toxoptera citricida Kirkaldy, atingiram a fase adulta e apresentaram 70 e 95\% de sobrevivência, respectivamente (Michaud, 2000). Segundo Abdel-Salam \& Abdel-Baky (2001), ovos de Sitotroga cerealella são uma dieta adequada para a criação desse coccinelídeo. $H$. axyridis, alimentada com o pulgão

Pesq. agropec. bras., Brasília, v.44, n.6, p.554-560, jun. 2009 
Aphis gossypii (Glover), atingiu desenvolvimento larval em oito dias; no entanto, quando alimentada exclusivamente com Brevicoryne brassicae (L.) ou com Megoura viciae Buckton, a joaninha não completou seu desenvolvimento, provavelmente em razão do efeito tóxico das presas (Tsaganou et al., 2004).

Apesar de ter sido introduzida em vários países como agente de controle biológico de pulgões, a joaninhaasiática pode ocasionar decréscimo nas populações de outros coccinelídeos, em razão da competição e da predação intraguilda (Koch, 2003; Cottrell, 2005; Koch et al., 2006). Avaliações sobre a sua competição com outros coccinelídeos, em laboratório, foram realizadas por Kajita et al. (2000), Cottrell (2005), Pell et al. (2008) e Ware \& Majerus (2008), que verificaram que sua atuação foi dominante em relação à Adalia bipunctata (L.), Coleomegilla maculata De Geer, Olla v-nigrum (Mulsant) e Propylaea japonica Thunberg, em razão do maior tamanho e da presença de estruturas protetoras como os espinhos dorsais ao longo do corpo das larvas.

$H$. axyridis pode atuar como predador intraguilda e ocasionar o deslocamento de coccinelídeos nativos (Koch, 2003). A predação intraguilda ocorre quando uma das duas espécies que competem pela mesma presa também consome seu competidor e influencia a estrutura da comunidade (Pell et al., 2008). De acordo com Michaud (2002), após a introdução de $H$. axyridis em pomares de citros na Flórida, a sua população aumentou, enquanto a população da joaninha dominante (Cycloneda sanguinea L.) declinou. A espécie asiática apresentou maior fecundidade e fertilidade e menor taxa de canibalismo larval, quando comparada à $C$. sanguinea. Em testes de laboratório para avaliar a predação intraguilda entre essas espécies, as larvas de $H$. axyridis foram mais agressivas ao atacar e se alimentar das larvas de C. sanguinea.

No Brasil, apesar de a presença da joaninha-asiática ter sido registrada em 2002 (Almeida \& Silva, 2002), existem poucas informações sobre sua biologia e preferência alimentar, e sobre a competição com outros coccinelídeos. Milléo et al. (2008) avaliaram a flutuação populacional dessa joaninha, em plantas frutíferas, e sua influência sobre outros coccinelídeos predadores, durante dois anos, e verificaram que sua população aumentou, enquanto a de outros coccinelídeos se reduziu do primeiro para o segundo ano.
Estudos sobre o desenvolvimento e reprodução desse inimigo natural, alimentado com diferentes dietas, e o seu comportamento em relação a outros predadores afidófagos devem ser realizados no Brasil para o conhecimento sobre o seu potencial como agente de controle biológico e sua competição com outros coccinelídeos.

O objetivo deste trabalho foi determinar os aspectos biológicos de $H$. axyridis, alimentada com duas espécies de presas, e avaliar a ocorrência de predação intraguilda em relação a Eriopis connexa (Germar).

\section{Material e Métodos}

O trabalho foi realizado no Laboratório de Entomologia, da Agência Paulista de Tecnologia dos Agronegócios (APTA), em Andradina, SP, de dezembro de 2006 a setembro de 2007.

Adultos e ninfas de Schizaphis graminum (Rondani) foram coletados em cultura de sorgo, da área experimental da APTA, e mantidos em caules de sorgo imersos em água, em copos de plástico de $200 \mathrm{~mL}$, com a extremidade superior vedada com filme de polietileno. Os pulgões foram transferidos para novos caules duas vezes por semana. A criação dos pulgões foi mantida a $25 \pm 1^{\circ} \mathrm{C}$ e umidade relativa de $70 \pm 10 \%$.

Cinco casais de $H$. axyridis e de E. connexa, oriundos da criação massal do Laboratório de Entomologia da APTA, foram mantidos em gaiolas de PVC de $10 \mathrm{~cm}$ de altura x $10 \mathrm{~cm}$ de diâmetro, revestidas internamente com papel sulfite para a obtenção dos ovos e com as extremidades vedadas por tecido "voile". Diariamente, as posturas foram coletadas e transferidas para placas de Petri de $9 \mathrm{~cm}$ de diâmetro, forradas com disco de papel sulfite, para a obtenção das larvas. Os adultos dos coccinelídeos foram alimentados com ovos de Anagasta kuehniella (Zeller), adquiridos da Empresa Bug Agentes Biológicos, e levedo de cerveja e mel na proporção de 2:1, pincelados em tiras de parafilme. A água foi fornecida por meio de algodão embebido e acondicionado em frasco de $10 \mathrm{~mL}$.

Larvas de $H$. axyridis recém-eclodidas foram individualizadas em tubos de vidro de $8 \mathrm{~cm}$ de altura por $2,5 \mathrm{~cm}$ de diâmetro, mantidas a $27 \pm 1^{\circ} \mathrm{C}$, em fotófase de 12 horas, à umidade relativa de $50 \pm 10 \%$, e alimentadas diariamente com ninfas e adultos do pulgão S. graminum ou ovos de A. kuehniella, que constituíram dois tratamentos, com 30 repetições cada um. As duas espécies de presas foram utilizadas 
em razão da facilidade de sua criação e obtenção. Diariamente, foram avaliados os parâmetros duração e viabilidade de cada instar e dos períodos de larva, pupa e larva-adulto, e o peso de larvas aos três e seis dias de vida.

Após a emergência, adultos de $H$. axyridis, originados de larvas alimentadas com cada espécie de presa, foram separados em dez casais, e cada par foi mantido em recipiente de plástico transparente de $300 \mathrm{~mL}$, vedado por tecido "voile". Os casais foram alimentados diariamente com ovos de A. kuehniella ou com S. graminum. Foram avaliados os parâmetros: peso dos adultos após 24 horas da emergência, duração dos períodos de pré-oviposição, oviposição e pós-oviposição, fertilidade diária e total e longevidade de adultos.

O delineamento utilizado para as avaliações dos parâmetros relacionados às fases jovem e adulta do coccinelídeo foi o inteiramente casualizado. Os dados foram submetidos à análise de variância, e as médias foram comparadas pelo teste de Tukey, a 5\% de probabilidade. Os dados relativos à sobrevivência foram transformados em arc sen $(x+0,5)^{0,5}$.

Para a avaliação da predação entre $H$. axyridis e E. connexa, larvas recém-eclodidas de cada espécie foram individualizadas em tubos de vidro de $2,5 \mathrm{~cm}$ de diâmetro x 8,5 cm de altura, e alimentadas à vontade com ovos de A. kuehniella até atingirem o quarto instar. Vinte e quatro horas após atingirem esse estádio, as larvas permaneceram sem suprimento de alimento por seis horas. Após o período de jejum, uma larva de quarto instar de cada espécie de predador foi mantida em placa de Petri de $9 \mathrm{~cm}$ de diâmetro, em presença ou ausência de ovos de A. kuehniella e de abrigo, que consistiu de secções de folha de papel sulfite sanfonada. Os tratamentos constituíram-se de: larvas sem suprimento de presa e de abrigo, sem suprimento de presa e com abrigo, com suprimento de presa e sem abrigo e com suprimento de presa e de abrigo. O delineamento utilizado foi o inteiramente casualizado, com dez repetições para cada tratamento. Os dados relacionados à taxa de predação entre as joaninhas foram transformados em arc sen $(x+0,5)^{0,5}$ e submetidos à análise de variância, e as médias foram comparadas pelo teste de Tukey, a $1 \%$ de probabilidade.

A avaliação da predação foi realizada 24 horas após o início do experimento, tendo-se considerado canibalismo quando ocorreu total alimentação de um predador sobre o outro ou quando os remanescentes do predador foram muito pequenos. A mortalidade por inanição foi avaliada de acordo com Phoofolo \& Obrycki (1998).

\section{Resultados e Discussão}

A duração do primeiro e segundo instares foi menor em larvas de $H$. axyridis alimentadas com $S$. graminum, em comparação àquelas criadas com ovos de A. kuehniella. No entanto, a espécie de presa não influenciou a duração dos demais instares do predador (Tabela 1).

A duração de cada instar, quando o coccinelídeo foi criado com ovos de A. kuehniella ou S. graminum, foi similar à observada por LaMana \& Miller (1998) e Lanzoni et al. (2004). Esses autores registraram valores de 2,5, 1,5, 1,8 e 4,4 e 2,3, 1,5, 2,0 e 4,7 dias, respectivamente, para o primeiro, segundo, terceiro e quarto instares a 26 e $25^{\circ} \mathrm{C}$, quando a espécie foi alimentada com Acyrthosiphon pisum (Harris) e Myzus persicae Sulzer.

O coccinelídeo apresentou taxas de desenvolvimento similares, mesmo quando alimentado com outras espécies de presas, provavelmente em razão de ter hábito polífago. Assim, o coccinelídeo completa o desenvolvimento de cada estádio imaturo em período de tempo semelhante, mesmo ao ser suprido com diferentes tipos de presas como dieta, desde que essas satisfaçam ao requerimento nutricional do predador.

A fase larval foi completada em período de tempo menor quando a joaninha foi suprida com o pulgão $S$. graminum do que quando suprida com ovos de A. kuehniella (Tabela 1). Os coccinelídeos apresentam hábito polífago, mas têm preferência por pulgões (Hodek \& Honek, 1996). Provavelmente, em consequência desse comportamento, o predador apresentou melhor taxa de desenvolvimento ao se alimentar de S. graminum, em comparação àquelas larvas criadas com ovos de A. kuehniella. A duração da fase larval registrada no presente trabalho, por sua vez, também foi menor do que aquela observada por Tsaganou et al. (2004) para essa espécie de coccinelídeo alimentado com pulgão A. gossypii (15,9 dias). As discrepâncias entre esses resultados possivelmente se relacionam às diferentes temperaturas utilizadas nos estudos e à quantidade, qualidade nutricional, tamanho e espécie de pulgão utilizada como dieta para o coccinelídeo. 
A duração do estágio pupal não foi influenciada pela espécie de presa oferecida como alimento (Tabela 1). O valor encontrado (quatro dias) foi próximo daqueles registrados por Saini et al. (2004).

O período larva-adulto durou aproximadamente dois dias a menos, quando o coccinelídeo foi alimentado com S. graminum, em comparação àquelas larvas criadas com ovos de A. kuehniella (Tabela 1). Abdel-Salam \& Abdel-Baky (2001) verificaram duração de 18,8 e 22,5 dias para $H$. axyridis criada a $27 \pm 1^{\circ} \mathrm{C}$, respectivamente, com ovos frescos ou congelados de $S$. cerealella. As diferenças registradas entre os trabalhos podem ser atribuídas à espécie de presa utilizada como alimento pela joaninha, já que a temperatura foi a mesma nos dois estudos. Esse resultado evidencia que o inimigo natural apresenta melhor taxa de desenvolvimento ao se alimentar de pulgões, por ser preferencialmente afidófago.

A sobrevivência durante as fases de desenvolvimento da joaninha, criada com as diferentes espécies de presas, variou de 70 a 100\% (Tabela 1). Das larvas alimentadas com ovos de A. kuehniella e S. graminum, 70 e $86,7 \%$, respectivamente, atingiram a fase adulta. De acordo com determinações de Michaud (2000), H. axyridis ao ser suprida com os pulgões $A$. spiraecola e $T$. citricida, proporcionou, respectivamente, que 70 e
95\% das larvas atingissem a fase adulta. Mignault et al. (2006) observaram que essa espécie de coccinelídeo, ao ser criada com o pulgão Aphis glycines Matsumura, apresentou 97,9\% de sobrevivência. Berkvens et al. (2008a) criaram essa joaninha com três tipos de dietas e observaram que 48, 90 e 98\% das larvas atingiram a fase adulta ao se alimentarem, respectivamente, de dietas com apenas pólen, ovos de Ephestia kuehniella Zeller e mistura de pólen com ovos de E. kuehniella.

As diferenças observadas na duração e sobrevivência das fases de desenvolvimento de $H$. axyridis, em relação às duas espécies de presas utilizadas no presente trabalho, ressaltam a influência das dietas sobre os seus estágios imaturos. O período de desenvolvimento e a sobrevivência da joaninha-asiática são extremamente influenciados pela qualidade do alimento (Pervez \& Omkar, 2006). De acordo com esses autores, o período de desenvolvimento diminui com dietas de alta qualidade nutricional.

O peso médio das larvas, aos três e seis dias de vida, e o dos adultos após 24 horas da emergência foi de 2,81, 12,18 e 28,81 $\mathrm{mg}$, respectivamente, quando as larvas foram alimentadas com ovos de A. kuehniella, e 7,64, 21,18 e 23,09 mg, respectivamente, quando o alimento foi $S$. graminum. As larvas alimentadas com ovos de A. kuehniella apresentaram menor peso aos três

Tabela 1. Duração (dias) e sobrevivência (\%), nas fases de desenvolvimento, de Harmonia axyridis alimentada com duas espécies de presa $\left(27 \pm 1^{\circ} \mathrm{C} \text { e } 50 \pm 10 \% \text { UR }\right)^{(1)}$.

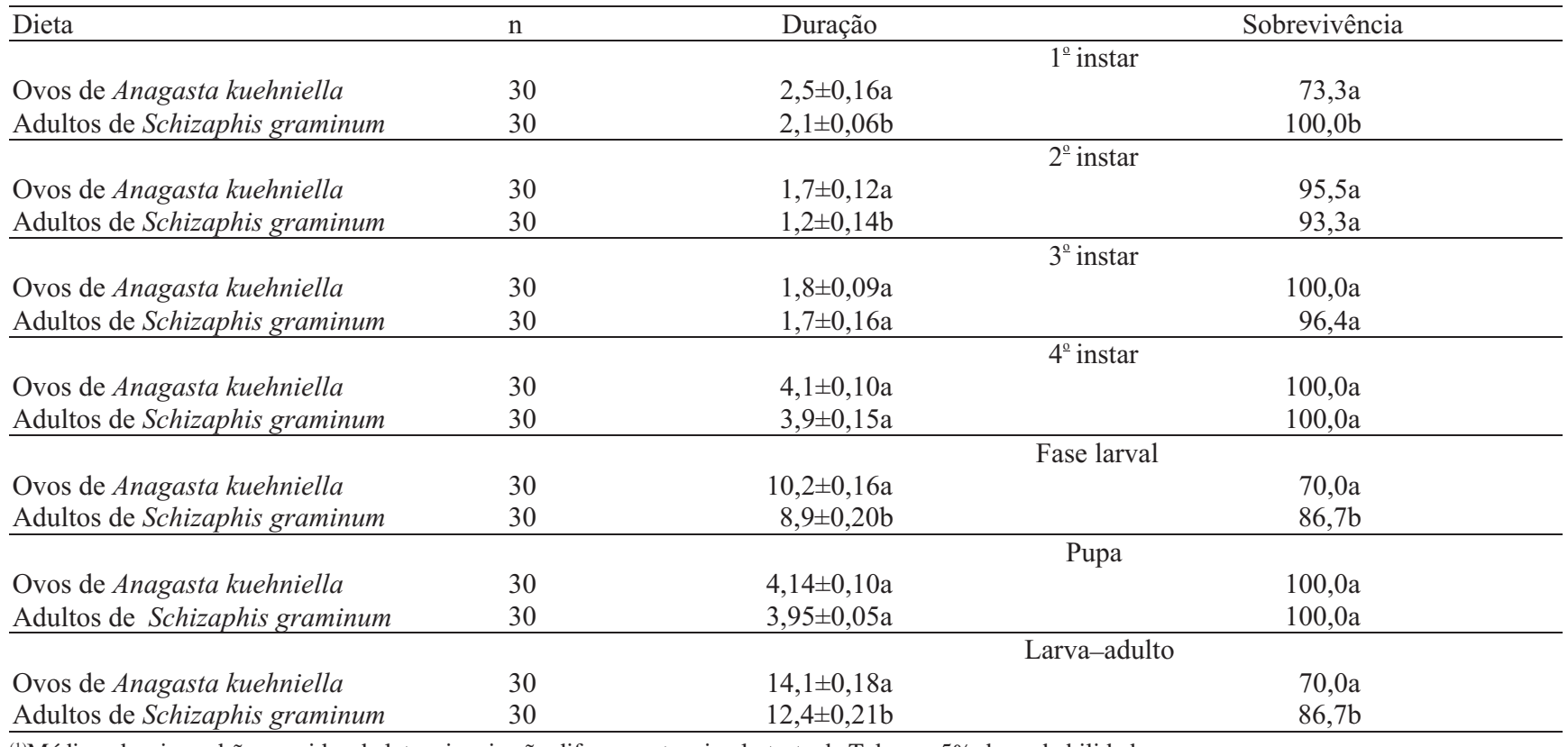

${ }^{(1)}$ Médias \pm desvio-padrão seguidas de letras iguais não diferem entre si pelo teste de Tukey, a 5\% de probabilidade. 
e seis dias de vida, no entanto, os adultos provenientes dessas larvas apresentaram peso maior do que aqueles de larvas alimentadas com S. graminum. Isso ocorreu, possivelmente, pelo fato de a duração do período larval de $H$. axyridis ter sido prolongada, em consequência da alimentação com ovos de A. kuehniella (Tabela 1). Para atingir o requerimento mínimo de nutrientes para seu desenvolvimento, o predador consumiu presas por período maior. Ao ingerir maior quantidade de biomassa, cresceu mais e teve incremento no peso.

Não foram observadas diferenças significativas nos parâmetros analisados, durante a fase adulta do coccinelídeo, quando alimentado com as duas espécies de presas (Tabela 2). O período de pré-oviposição do coccinelídeo, alimentado com ovos de A. kuehniella ou de $S$. graminum, foi maior do que aqueles observados por Abdel-Salam \& Abdel-Baky (2001) e Lanzoni et al. (2004), com $H$. axyridis suprida com ovos frescos de S. cerealella ( 8,1 dias) a $27 \pm 1^{\circ} \mathrm{C}$ e pulgão $M$. persicae (7,4 dias) a $25^{\circ} \mathrm{C}$, respectivamente.

Os períodos de oviposição de $H$. axyridis, alimentada comovos de A. kuehniella eS. graminum, foram próximos aos registrados por Abdel-Salam \& Abdel-Baky (2001) para essa espécie alimentada com ovos frescos de S. cerealella (49 dias). No entanto, esses períodos foram maiores do que os obtidos por Lanzoni et al. (2004), que forneceram M. persicae (13,7 dias), e menores do que os obtidos por Mignault et al. (2006), que forneceram A. glycines (103,2 dias) a $H$. axyridis. O período de pós-oviposição foi maior para ambos os tratamentos do que os obtidos por Abdel-Salam \& Abdel-Baky (2001) que criaram o coccinelídeo com ovos frescos (5,1 dias) e congelados de $S$. cerealella (6,8 dias).

A longevidade de fêmeas e machos variou de 67,3 a 76,2 dias (Tabela 2). Hodek (1973) e Iperti (1999) ressaltaram que a longevidade dos coccinelídeos é bastante variável e atinge de alguns meses a três anos.

Os números total e diário de ovos produzidos por $H$. axyridis, ao se alimentar com S. graminum e ovos de
A. kuehniella, diferiram de vários trabalhos (Tabela 2). Essa espécie de coccinelídeo alimentada com ovos de $S$. cerealella a $27^{\circ} \mathrm{C}$ colocou o total de 715,3 ovos e a média de 14,5 ovos por dia (Abdel-Salam \& Abdel-Baky, 2001). Lanzoni et al. (2004) observaram que a mesma espécie de predador, alimentada com o pulgão $M$. persicae a $25^{\circ} \mathrm{C}$, produziu o total de 560,5 ovos - média de 18,3 por dia. Mignault et al. (2006) relataram taxa de fecundidade de 2.008 ovos, quando a joaninha foi alimentada com A. glycines a $24^{\circ} \mathrm{C}$. Essas diferenças de fecundidade mencionadas podem estar relacionadas às diferentes espécies de presas e temperaturas empregadas nos estudos.

O pulgão $S$. graminum foi mais adequado como alimento a $H$. axyridis, pois as larvas alimentadas com essa presa completaram o desenvolvimento em menor tempo, apresentaram maior percentual de sobrevivência(Tabela1)emaiores pesos do que aquelas alimentadas com ovos de A. kuehniella. Entretanto, ambas as presas utilizadas no presente trabalho podem ser consideradas essenciais, de acordo com o sistema de classificação proposto por Hodek (1973), pois permitiram o desenvolvimento, a oviposição e a sobrevivência da joaninha. Estudos têm demonstrado que ovos de E. kuehniella, S. cerealella e A. kuehniella são eficientes dietas para a criação de Coccinellidae (Kato et al., 1999; Abdel-Salam \& Abdel-Baky, 2001; Berkvens et al., 2008b). No presente trabalho, a dieta com ovos de A. kuehniella foi adequada para criação de $H$. axyridis. Essa espécie de presa poderá ser utilizada na criação de $H$. axyridis em laboratório, como alternativa ao pulgão.

Não foi observada predação das larvas de $H$. axyridis por E. connexa, em nenhum dos tratamentos. Possivelmente, larvas de $H$. axyridis são favorecidas, por apresentar espinhos dorsais ao longo do corpo e maior tamanho do que as de E. connexa. De acordo com Pell et al. (2008), as características-chave que favorecem a joaninha-asiática, na competição intraguilda, são: o maior tamanho, comportamento

Tabela 2. Longevidade (dias) e fecundidade de fêmeas de Harmonia axyridis, alimentadas com duas espécies de presa $\left(27 \pm 1^{\circ} \mathrm{C} \text { e } 50 \pm 10 \% \text { UR }\right)^{(1)}$.

\begin{tabular}{|c|c|c|c|c|c|c|}
\hline$\overline{\text { Dieta }}$ & Pré-oviposição & Oviposição & Pós-oviposição & Longevidade $^{(2)}$ & $\mathrm{N}^{\mathrm{o}}$ total de ovos & $\begin{array}{c}\mathrm{N}^{\mathrm{o}} \text { de ovos } \\
\text { por dia }\end{array}$ \\
\hline Ovos de Anagasta kuehniella & $9,8 \pm 1,03 \mathrm{a}$ & $47,3 \pm 2,79 \mathrm{a}$ & $17,0 \pm 3,33 a$ & $74,1 \pm 4,53 a$ & $887,6 \pm 78,07 a$ & $18,8 \pm 1,30 \mathrm{a}$ \\
\hline Adultos de Schizaphis graminum & $10,6 \pm 0,83 \mathrm{a}$ & $51,7 \pm 1,52 \mathrm{a}$ & $13,9 \pm 3,73 a$ & $76,2 \pm 4,00 \mathrm{a}$ & $822,5 \pm 46,04 \mathrm{a}$ & $15,9 \pm 0,92 \mathrm{a}$ \\
\hline
\end{tabular}


agressivo, a extrema polifagia e o fato de apresentar estratégias físicas e químicas de defesa.

Ware \& Majerus (2008) avaliaram a predação entre $H$. axyridis e 12 espécies de coccinelídeos e demonstraram que a joaninha-asiática foi o predador intraguilda de quase todas as espécies, com exceção de Anatis ocellata (L.). Segundo os autores, as larvas de $A$. ocellata são maiores que as de $H$. axyridis e apresentam espinhos dorsais, e tais características favoreceram a espécie em ser pouco atacada por H. axyridis.

As taxas de predação ou mortalidade de larvas de $E$. connexa por $H$. axyridis foram influenciadas pela disponibilidade de presa e de abrigo $(\mathrm{F}=14 ; \mathrm{p}<0,01$; $\mathrm{CV}=35 \%)$. Nos tratamentos com suprimento de ovos de A. kuehniella, na presença e na ausência de refúgio, essas taxas foram de 20 e $60 \%$, respectivamente. Quando as larvas dos coccinelídeos não tiveram suprimento de presa, as taxas de predação de E. connexa por $H$. axyridis foram de $40 \%$ na presença de refúgio e de $100 \%$ na ausência deste.

Cottrell (2005), ao avaliar a taxa de canibalismo e predação de ovos de joaninhas pelos adultos de H. axyridis, verificou que Coleomegilla maculata De Geer e Olla v-nigrum (Mulsant) apresentaram maior número de ovos predados por $H$. axyridis. No entanto, esse autor observou que, ao se adicionar uma fonte alternativa de alimento, as taxas de predação e canibalismo foram reduzidas.

A predação intraguilda dependeu, principalmente, da disponibilidade de presa. O refúgio também influenciou a interação entre as duas espécies e diminuiu a taxa de predação de $H$. axyridis sobre E. connexa, em presença ou em ausência de alimento. Esses resultados concordam com os de Kajita et al. (2000). Esses autores avaliaram a interação entre Adalia bipunctata (L.) e H. axyridis, em várias densidades de Aphis craccivora Koch, e não observaram predação de $H$. axyridis por A. bipunctata, quando submetidas a altas densidades de presas. A taxa de predação de A. bipunctata por H. axyridis foi de $25 \%$ em condições de alta densidade de pulgões, $60 \%$ com suprimento de 50 pulgões e $75 \%$ sem pulgões.

H. axyridis demonstrou ser dominante na competição com E. connexa, em condições de laboratório. Provavelmente, em casa de vegetação e campo, esse comportamento se repetirá, pois conforme De Clercq et al. (2003), em programas de controle biológico, vários predadores generalistas são utilizados simultaneamente, para controlar um complexo de pragas que ocorrem em determinado agroecossistema. Nesse caso, a ocorrência de predação intraguilda poderá ocasionar decréscimo na taxa de predação do insetopraga e impedir o sucesso do programa, pelo fato de o predador se alimentar de outro inimigo natural (Mallampalli et al., 2002).

\section{Conclusões}

1. Ovos de Anagasta kuehniella e o pulgão Schizaphis graminum são presas essenciais para Harmonia axyridis.

2. H. axyridis é a espécie dominante na competição com Eriopis connexa e atua como predador intraguilda.

\section{Referências}

ABDEL-SALAM, A.H.; ABDEL-BAKY, N.F. Life table and biological studies of Harmonia axyridis Pallas (Col., Coccinellidae) reared on the grain moth eggs of Sitotroga cerealella Olivier (Lep., Gelechiidae). Journal of Applied Entomology, v.125, p.455-462, 2001.

ALMEIDA, L.M. de; SILVA, V.B. da. First record of Harmonia axyridis (Pallas) (Coleoptera: Coccinellidae): a lady beetle native to the Palaearctic region. Revista Brasileira de Zoologia, v.19, p.941-944, 2002.

BERKVENS, N.; BONTE, J.; BERKVENS, D.; DEFORCE, K.; TIRRY, L.; DE CLERCQ, P. Pollen as an alternative food for Harmonia axyridis. In: ROY, H.E.; WAJNBERG, E. (Ed.). From biological control to invasion: the ladybird Harmonia axyridis as a model species. The Netherlands: Springer, 2008a. p.201-210.

BERKVENS, N.; BONTE, J.; BERKVENS, D.; TIRRY, L.; DE CLERCQ, P. Influence of diet and photoperiod on development and reproduction of European populations of Harmonia axyridis (Pallas) (Coleoptera: Coccinellidae). In: ROY, H.E.; WAJNBERG, E. (Ed.). From biological control to invasion: the ladybird Harmonia axyridis as a model species. The Netherlands: Springer, 2008b. p.211-221.

BROWN, M.W.; MILLER, S.S. Coccinellidae (Coleoptera) in apple orchards of eastern West Virginia and the impact of invasion by Harmonia axyridis. Entomological News, v.109, p.143-151, 1998.

COTTRELL, T.E. Predation and cannibalism of lady beetle eggs by adult lady beetles. Biological Control, v.34, p.159-164, 2005.

DE CLERCQ, P.; PEETERS, I.; VERGAUWE, G.; THAS, O. Interaction between Podisus maculiventris and Harmonia axyridis, two predators used in augmentative biological control in greenhouse crops. BioControl, v.48, p.39-55, 2003.

HODEK, I. Life history and biological properties. In: HODEK, I. Biology of Coccinellidae. Prague: Academy of Sciences, 1973. p.70-75. 
HODEK, I.; HONEK A. Ecology of Coccinellidae. Dordrecht: Kluwer Academic, 1996. 464p.

IPERTI, G. Biodiversity of predaceous Coccinellidae in relation to bioindication and economic importance. Agriculture, Ecosystems and Environment, v.74, p.323-342, 1999.

KAJITA, K.; TAKANO, F.; YASUDA, H.; AGARWALA, B.K. Effects of indigenous ladybird species (Coleoptera: Coccinellidae) on the survival of an exotic species in relation to prey abundance. Applied Entomology and Zoology, v.35, p.473-479, 2000.

KATO, C.M.; BUENO, V.H.P.; MORAES, J.C.; AUAD, A.M. Criação de Hippodamia convergens Guérin-Meneville (Coleoptera: Coccinellidae) em ovos de Anagasta kuehniella (Zeller) (Lepidoptera: Pyralidae). Anais da Sociedade Entomológica do Brasil, v.28, p.455-459, 1999.

KOCH, R.L. The multicolored Asian lady beetle, Harmonia axyridis: a review of its biology, uses in biological control, and non-target impacts. Journal of Insect Science, v.3, p.1-16, 2003.

KOCH, R.L.; VENETTE, R.C.; HUTCHISON, W.D. Invasions by Harmonia axyridis (Pallas) (Coleoptera: Coccinellidae) in the Western Hemisphere: implications for South America. Neotropical Entomology, v.35, p.421-434, 2006.

KURODA, T.; MIURA, K. Comparison of the effectiveness of two methods for releasing Harmonia axyridis (Pallas) (Coleoptera: Coccinellidae) against Aphis gossypii Glover (Homoptera: Aphididae) on cucumbers in a greenhouse. Applied Entomology and Zoology, v.38, p.271-274, 2003.

LAMANA, M.L.; MILLER, J.C. Temperature-dependent development in an Oregon population of Harmonia axyridis (Coleoptera: Coccinellidae). Environmental Entomology, v.27, p.1001-1005, 1998.

LANDIS, D.A.; FOX, T.B.; COSTAMAGNA, A.C. Impact of multicolored Asian lady beetle as a biological control agent. American Naturalist, v.50, p.153-154, 2004.

LANZONI, A.; ACCINELLI, G.; BAZZOCCHI, G.G.; BURGIO, G. Biological traits and life table of the exotic Harmonia axyridis compared with Hippodamia variegata and Adalia bipunctata (Col.: Coccinellidae). Journal of Applied Entomology, v.128, p.298-306, 2004.

MALLAMPALLI, N.; CASTELLANOS, I.; BARBOSA, P. Evidence for intraguild predation by Podisus maculiventris on a ladybeetle, Coleomegilla maculata: implications for biological control of Colorado potato beetle, Leptinotarsa decemlineata. BioControl, v.47, p.387-398, 2002.
MICHAUD, J.P. Development and reproduction of ladybeetles (Coleoptera: Coccinellidae) on the citrus aphids Aphis spiraecola Patch and Toxoptera citricida (Kirkaldy) (Homoptera: Aphididae). Biological Control, v.18, p.287-297, 2000.

MICHAUD, J.P. Invasion of the Florida citrus ecosystem by Harmonia axyridis (Coleoptera: Coccinellidae) and asymmetric competition with a native species, Cycloneda sanguinea. Environmental Entomology, v.31, p.827-835, 2002.

MIGNAULT, M.P.; ROY, M.; BRODEUR, J. Soybean aphid predators in Quebec and the suitability of Aphis glycines as prey for three Coccinellidae. BioControl, v.51, 89-106, 2006.

MILLÉO, J.; SOUZA, J.M.T. de; BARBOLA, I. de F.; HUSCH, P.E. Harmonia axyridis em árvores frutíferas e impacto sobre outros coccinelídeos predadores. Pesquisa Agropecuária Brasileira, v.43, p.537-540, 2008.

PELL, J.K.; BAVERSTOCK, J.; ROY, H.E; WARE, R.L.; MAJERUS, M.E.N. Intraguild predation involving Harmonia axyridis: a review of current knowledge and future perspectives. BioControl, v.53, p.147-168, 2008.

PERVEZ, A.; OMKAR. Ecology and biological control application of multicoloured Asian ladybird, Harmonia axyridis: a review. Biocontrol Science and Technology, v.16, p.111-128, 2006.

PHOOFOLO, M.W.; OBRYCKI, J.J. Potential for intraguild predation and competition among predatory Coccinellidae and Chrysopidae. Entomologia Experimentalis et Applicata, v.89, p.47-55, 1998.

SAINI, E.D. Presencia de Harmonia axyridis (Pallas) (Coleoptera: Coccinellidae) en la provincia de Buenos Aires: aspectos biológicos y morfológicos. Revista de Investigaciones Agropecuarias, v.33, p.151-160, 2004.

TSAGANOU, F.C.; HODGSON, C.J.; ATHANASSIOU, C.G.; KAVALLIERATOS, N.G.; TOMANOVIC, Z. Effect of Aphis gossypii Glover, Brevicoryne brassicae (L.), and Megoura viciae Buckton (Hemiptera: Aphidoidea) on the development of the predator Harmonia axyridis (Pallas) (Coleoptera: Coccinellidae). Biological Control, v.31, p.138-144, 2004.

WARE, R.L.; MAJERUS, M.E.N. Intraguild predation of immature stages of British and Japanese coccinellids by the invasive ladybird Harmonia axyridis. BioControl, v.53, p.169-188, 2008.

Recebido em 5 de dezembro de 2008 e aprovado em 29 de maio de 2009 